\title{
LINEAR TRANSFORMATIONS ON MATRICES
}

\author{
BY \\ D. ž. DJOKOVIĆ(')
}

\begin{abstract}
The real orthogonal group $O(n)$, the unitary group $U(n)$ and the symplectic group $\mathrm{Sp}(n)$ are embedded in a standard way in the real vector space of $n \times n$ real, complex and quaternionic matrices, respectively. Let $F$ be a nonsingular real linear transformation of the ambient space of matrices such that $F(G) \subset G$ where $G$ is one of the groups mentioned above. Then we show that either $F(x)=a \sigma(x) b$ or $F(x)=a \sigma\left(x^{*}\right) b$ where $a, b \in G$ are fixed, $x^{*}$ is the transpose conjugate of the matrix $x$ and $\sigma$ is an automorphism of reals, complexes and quaternions, respectively.
\end{abstract}

1. Introduction. In his survey paper [4], M. Marcus has stated seven conjectures. In this paper we shall study the last two of these conjectures.

Let $D$ be one of the following real division algebras $\mathbf{R}$ (the reals), $\mathbf{C}$ (the complex numbers) or $\mathbf{H}^{\prime}$ (the quaternions). As usual we assume that $\mathbf{R} \subset \mathbf{C} \subset \mathbf{H}$. We denote by $M_{n}(D)$ the real algebra of all $n \times n$ matrices over $D$. If $\xi \in D$ we denote by $\bar{\xi}$ its conjugate in $D$. Let $A$ be the automorphism group of the real algebra $D$. If $D=\mathrm{R}$ then $A$ is trivial. If $D=\mathrm{C}$ then $A$ is cyclic of order 2 with conjugation as the only nontrivial automorphism. If $D=\mathbf{H}$ then the conjugation is not an isomorphism but an anti-isomorphism of $\mathbf{H}$. Every automorphism of $\mathbf{H}$ is inner (by the Noether-Skolem theorem) and every automorphism of $\mathbf{H}$ commutes with conjugation.

If $x \in M_{n}(D)$ then we let $\bar{x}$ be the conjugate matrix, ${ }^{t} x$ the transpose of $x$ and $x^{*}$ the conjugate transpose of $x$. The rule $(x y)^{*}=y^{*} x^{*}$ is valid for any $x, y \in M_{n}(D)$. If $x \in M_{n}(D)$ and $\sigma \in A$ we let $\sigma(x)$ be the matrix obtained from $x$ by applying $\sigma$ to each of its entries.

Let $e \in M_{n}(D)$ be the identity matrix and put $G=\left\{x \in M_{n}(D) \mid x x^{*}=e\right\}$. We have

$$
\begin{array}{cl}
G=O(n) \text { the real orthogonal group, } & \text { if } D=\mathbf{R} ; \\
G=U(n) \text { the unitary group, } & \text { if } D=\mathbf{C} ; \\
G=\operatorname{Sp}(n) \text { the symplectic group, } & \text { if } D=\mathbf{H} .
\end{array}
$$

These are classical compact Lie groups. When $D=\mathbf{R}$ then $G=O(n)$ has two components and its identity component is the so called special orthogonal group SO $(n)$, also called the rotation group.

Received by the editors December 19, 1972.

AMS (MOS) subject classifications (1970). Primary 20G25; Secondary 15A69, 22 C05.

Key words and phrases. Orthogonal, unitary and symplectic group, invariant subspace, tangent space, skew-hermitian matrices, Noether-Skolem theorem.

(1) This work was supported in part by NRC Grant A-5285. 
Now, we can state a slightly modified version of Conjecture 6:

Conjecture 6. Let $F: M_{n}(D) \rightarrow M_{n}(D)$ be an R-linear transformation such that $F(G) \subset G$. Then $F$ must have one of the following two forms: Either (1) $F(x)$ $=a \sigma(x) b$ for all $x \in M_{n}(D)$, or (2) $F(x)=a \sigma\left(x^{*}\right) b$ for all $x \in M_{n}(D)$, where $a, b \in G$ and $\sigma \in A$. Also, if $D=\mathrm{R}$ and $F(\mathrm{SO}(n)) \subset \mathrm{SO}(n)$ then (1) or (2) holds.

In his earlier paper [3] M. Marcus has shown that if $D=C$ and $F$ is also supposed to be a C-linear map then $F(G) \subset G$ implies that either

$$
\begin{array}{ll}
F(x)=a x b & \text { for all } x \in M_{n}(\mathbf{C}), \text { or } \\
F(x)=a^{t} x b & \text { for all } x \in M_{n}(\mathbf{C}),
\end{array}
$$

where $a, b \in U(n)$. These results agree with the conjecture because if we want $F$ to be $\mathbf{C}$-linear we must take $\sigma=$ identity in (1) and $\sigma=$ conjugation in (2).

If $D=\mathbf{H}$ note that $x \in \operatorname{Sp}(n)$ implies that $\sigma\left(x^{*}\right) \in \operatorname{Sp}(n)$ because $\sigma \in A$ commutes with the conjugation.

One can equip the real vector space $M_{n}(D)$ with a positive definite scalar product as follows:

$$
\langle x, y\rangle=\operatorname{Re} \operatorname{tr}\left(x y^{*}\right) .
$$

The Conjecture 6, as stated above, has been proved in [2] under additional assumption that $F$ is orthogonal when $M_{n}(D)$ is equipped with the scalar product (3).

Here we shall prove Conjecture 6 when $F$ is assumed to be nonsingular.

To introduce the next conjecture let $V$ be a finite dimensional complex Hilbert space and let $V \otimes_{\mathbf{C}} V$ be equipped with the scalar product which satisfies: $\langle x \otimes y, a \otimes b\rangle=\langle x, a\rangle\langle y, b\rangle$ for all $x, y, a, b$ in $V$.

Conjecture 7. If $u: V \otimes_{\mathrm{C}} V \rightarrow V \otimes_{\mathrm{C}} V$ is a complex linear map such that

$$
\|u(a \otimes b)\|=\|a \otimes b\| \text { for all } a, b \in V
$$

then $u$ must be unitary.

We shall show that this conjecture is true. It is worthwhile to remark that the analogous conjecture for real Hilbert spaces is false. I am indebted to M. Marcus for a counterexample to the latter.

2. Matrix equation $a x=x a^{*}$.

Theorem 1. Let $a \in O(n)$ have the eigenvalues

$$
\begin{aligned}
& \exp \left( \pm i \theta_{r}\right) \text { of multiplicity } k_{r}, 1 \leq r \leq s \\
& +1 \text { of multiplicity } p \\
& -1 \text { of multiplicity } q
\end{aligned}
$$


where $0<\theta_{r}<\pi$ are distinct, $p, q$, s are nonnegative integers and $2\left(k_{1}+\cdots+k_{s}\right)$ $+p+q=n$.

Then the real vector space of skew-symmetric matrices $x \in M_{n}(\mathbf{R})$ satisfying the equation

$$
a x=x^{\prime} a
$$

has dimension $\sum_{r=1}^{s} k_{r}\left(k_{r}-1\right)+\frac{1}{2} p(p-1)+\frac{1}{2} q(q-1)$.

Proof. By performing a similarity transformation by a suitable orthogonal matrix we can assume that $a$ has the canonical quasi-diagonal form. The diagonal blocks of $a$ are the matrices

$$
a_{r}=\left(\begin{array}{cc}
\cos \theta_{r} & -\sin \theta_{r} \\
\sin \theta_{r} & \cos \theta_{r}
\end{array}\right), \quad 1 \leq r \leq s
$$

each $a_{r}$ being repeated $k_{r}$ times, followed by $p$ one's and $q$ minus one's.

We partition the unknown skew-symmetric matrix $x$ into blocks of the same size as the corresponding blocks of $a$.

Now we make the following observations.

(i) If $y$ is a $2 \times 2$ diagonal block of $x$ corresponding to an $a_{r}$ then $y=0$. Indeed (4) implies that $a_{r} y=y^{\prime} a_{r}$. Since $y$ is skew-symmetric and $\sin \theta_{r} \neq 0$ we must have $y=0$.

(ii) If

$$
\left(\begin{array}{cc}
0 & y \\
-t y & 0
\end{array}\right)
$$

is a principal submatrix of $x$ with $y$ some $2 \times 2$ block of $x$ and if the corresponding submatrix of $a$ is

$$
\left(\begin{array}{cc}
a_{r} & 0 \\
0 & a_{m}
\end{array}\right), \quad r \neq m
$$

then $y=0$.

In this case (4) implies that $a_{r} y=y^{\prime} a_{m}$. Since $a_{r}$ and $a_{m}$ are not similar we see that $y$ must be singular. Hence we can write $y=\left(\begin{array}{c}\lambda_{\alpha \alpha} \lambda \beta \\ \mu \alpha \beta\end{array}\right)$ where $\lambda, \mu, \alpha, \beta \in \mathbf{R}$. By inspection of the first columns of $a_{r} y$ and $y^{t} a_{m}$ we find that

$$
\begin{aligned}
& \alpha(\lambda \cos \theta-\mu \sin \theta)=\lambda(\alpha \cos \phi-\beta \sin \phi), \\
& \alpha(\lambda \sin \theta+\mu \cos \theta)=\mu(\alpha \cos \phi-\beta \sin \phi)
\end{aligned}
$$

where $\theta_{r}=\theta, \theta_{m}=\phi$.

From (5) we deduce that $\alpha\left(\lambda \mu \cos \theta-\mu^{2} \sin \theta\right)=\alpha\left(\lambda^{2} \sin \theta+\lambda \mu \cos \theta\right)$ and then $\alpha\left(\lambda^{2}+\mu^{2}\right) \sin \theta=0$. But $\sin \theta \neq 0$. If $\lambda^{2}+\mu^{2}=0$ then $\lambda=\mu=0$ and $y=0$. Otherwise $\alpha=0$, then from (5) we see that $\beta=0$ and then $y=0$ follows. 
(iii) If

$$
\left(\begin{array}{cc}
0 & y \\
-y & 0
\end{array}\right)
$$

is a principal submatrix of $x$ where $y$ is a $2 \times 2$ block of $x$ and the corresponding submatrix of $a$ is

$$
\left(\begin{array}{cc}
a_{r} & 0 \\
0 & a_{r}
\end{array}\right)
$$

then the equation $a_{r} y=y^{\prime} a_{r}$ (which follows from (4)) is satisfied only by the matrices $y$ of the form $\left(\begin{array}{c}\alpha \beta \\ \beta\end{array}-\alpha\right)$. This can be seen by a simple computation.

(iv) If

$$
\left(\begin{array}{cc}
0 & y \\
-t y & 0
\end{array}\right)
$$

is a principal submatrix of $x$ where $y$ is a $2 \times 1$ block of $x$ and the corresponding submatrix of $a$ is

$$
\left(\begin{array}{cc}
a_{r} & 0 \\
0 & \pm 1
\end{array}\right)
$$

then $y=0$.

Indeed, (4) implies that $a_{r} y= \pm y$ but $a_{r}$ has no real eigenvalues.

(v) If $\left(\begin{array}{cc}0 & a \\ -\alpha & 0\end{array}\right)$ is a principal submatrix of $x$ and $\left(\begin{array}{cc}1 & 0 \\ 0 & -1\end{array}\right)$ is the corresponding submatrix of $a$ then $\alpha=0$.

This is immediate from (4).

After these observations the formula for the dimension of the space of skewsymmetric solutions of (4) follows by a simple counting.

Theorem 2. Let $a \in U(n)$ have the eigenvalues

$$
\begin{aligned}
& \exp \left(i \theta_{r}\right) \text { of multiplicity } k_{r}^{\prime} ; 1 \leq r \leq s, \\
& \exp \left(-i \theta_{r}\right) \text { of multiplicity } k_{r}^{\prime \prime} ; 1 \leq r \leq s, \\
& +1 \text { of multiplicity } p ; \\
& -1 \text { of multiplicity } q ;
\end{aligned}
$$

where $k_{r}^{\prime}, k_{r}^{\prime \prime}, p, q, s$ are nonnegative integers, the $\theta_{r}^{\prime}$ 's are distinct and lie in the interval $0<\theta<\pi$, and $\sum_{r=1}^{s}\left(k_{r}^{\prime}+k_{r}^{\prime \prime}\right)+p+q=n$.

Then the real vector space of skew-hermitian matrices $x \in M_{n}(C)$ satisfying the equation $a x=x a^{*}$ has dimension $2 \sum_{r=1}^{s} k_{r}^{\prime} k_{r}^{\prime \prime}+p^{2}+q^{2}$.

Proof. We can assume that $a$ has diagonal form. It is easy to verify that the $(r, m)$-entry of $x$ must be 0 if the $r$ th and $m$ th diagonal entry of $a$ are not 
conjugate; otherwise this entry may be arbitrary if $r \neq m$ and arbitrary purely imaginary number if $r=m$.

Then a simple counting will complete the proof.

Before stating the next theorem we need to introduce some more terminology. We say that two quaternions $\xi$ and $\eta$ are similar if there exists $\sigma \in A$ such that $\sigma(\xi)=\eta$. It follows easily from the Noether-Skolem theorem (see [1]) that $\xi$ and $\bar{\xi}$ are always similar. It is well known that the eigenvalues of $u \in \mathrm{Sp}(n)$ are determined uniquely up to similarity.

Theorem 3. Let $a \in \mathrm{Sp}(n)$ have the eigenvalues

$$
\begin{aligned}
& \lambda_{r} \text { with multiplicity } k_{r}, 1 \leq r \leq s ; \\
& +1 \text { with multiplicity } p, \\
& -1 \text { with multiplicity } q,
\end{aligned}
$$

where $\lambda_{r} \in \mathbf{H}$ are nonsimilar and not real; $p, q, s$ are nonnegative integers and $\left(k_{1}+\cdots+k_{s}\right)+p+q=n$. Then the real vector space of skew-hermitian matrices $x \in M_{n}(\mathbf{H})$ satisfying the equation

$$
a x=x a^{*}
$$

has the dimension $\sum_{r=1}^{s} k_{r}\left(k_{r}+1\right)+p(2 p+1)+q(2 q+1)$.

Proof. By performing a similarity transformation by a suitable sympletic matrix we can assume that $a$ has diagonal form with the above listed eigenvalues on the diagonal.

Let

$$
\left(\begin{array}{cc}
\alpha & \beta \\
-\bar{\beta} & \gamma
\end{array}\right), \quad \alpha+\bar{\alpha}=\gamma+\bar{\gamma}=0
$$

be a principal submatrix of $x$ and

$$
\left(\begin{array}{cc}
\lambda_{r} & 0 \\
0 & \lambda_{m}
\end{array}\right)
$$

the corresponding submatrix of $a$. If $r \neq m$ we claim that $\beta=0$. Indeed, (6) gives

$$
\lambda_{r} \beta=\beta \bar{\lambda}_{m} .
$$

Since $\lambda_{r}$ and $\bar{\lambda}_{m}$ are not similar we must have $\beta=0$.

If $r=m$ we claim that the solution space of Equation (7) has dimension 2. Indeed, we can assume that $\lambda_{r}=i$ is one of the basic units of $\mathbf{H}$ and then our assertion is obvious because the solution space is spanned by the other two basic units $j$ and $k$, say. 
For $\alpha$ we have the equation $\lambda_{r} \alpha=\alpha \bar{\lambda}_{r}$ and again the space of solutions has dimension 2.

Similarly, if the corresponding submatrix of $a$ is

$$
\left(\begin{array}{cc}
\lambda, & 0 \\
0 & \pm 1
\end{array}\right) \text { or }\left(\begin{array}{cc}
1 & 0 \\
0 & -1
\end{array}\right)
$$

we get again $\beta=0$.

Finally, if the corresponding submatrix of $a$ is $\pm\left(\begin{array}{ll}1 & 0 \\ 0 & 1\end{array}\right)$ then $\beta$ may be arbitrary and $\alpha$ and $\gamma$ are subject only to the condition $\alpha+\bar{\alpha}=\gamma+\bar{\gamma}=0$.

Thus $x$ must be a quasi-diagonal matrix with diagonal blocks corresponding to the blocks of equal diagonal entries of $a$.

Simple counting gives us the required formula for the dimension.

3. Conjecture 6 (General case). From now on we shall assume that $F$ is nonsingular and satisfies $F(G) \subset G$. Without loss of generality we can assume that $F(e)=e$. Indeed, if $F(e)=a$ then it suffices to replace $F$ by $F_{1}$ which is defined by $F_{1}(x)=a^{-1} F(x)$ since then $F_{1}(e)=e$.

We consider $G$ as an analytic submanifold of $M_{n}(D)$. As usual, we shall identify the tangent vectors of $G$ at some point of $G$ with the corresponding matrices in $M_{n}(D)$. In this sense we can say that the tangent space to $G$ at $e$ is the real vector space of skew-hermitian matrices in $M_{n}(D)$. Let us denote this vector space by $S$, i.e., $S=\left\{x \in M_{n}(D) \mid x^{*}=-x\right\}$. Then the tangent space to $G$ at some point $a \in G$ will be the space $a \cdot S=\{a \cdot x \mid x \in S\}$. Since $F$ is linear it coincides with its own differential. Thus $F$ must map the tangent space of $G$ at $a \in G$ bijectively to the tangent space of $G$ at $F(a)$, i.e., we have

$$
F(a \cdot S)=F(a) \cdot S, \text { for all } a \in G .
$$

For $a=e$ we get $F(S)=S$ because $F(e)=e$.

We have $F(a \cdot S \cap S)=F(a) \cdot S \cap S$ for $a \in G$ and in particular,

$$
\operatorname{dim}(F(a) \cdot S \cap S)=\operatorname{dim}(a \cdot S \cap S), \quad a \in G .
$$

It is easy to see that $a S \cap S$ is precisely the space whose dimension was calculated in $\$ 2$.

It is easy to verify Conjecture 6 when $n=1$. Indeed, the case $D=\mathbf{R}$ is trivial. If $D=C$ then we identify $G$ with the unit circle in C. Since $F(G)=G$ and $F(1)=1$ we see that $F$ is orthogonal and so either it is identity or conjugation. If $D=\mathbf{H}$ then $G=\mathrm{Sp}$ (1) can be identified with the unit 3-sphere of $\mathbf{H}$. Since $F(G)=G$ it follows that $F$ is orthogonal. From $F(1)=1$ it follows that $F$ preserves the pure quaternions. Since $A$ is transitive on the unit sphere of the space of pure quaternions we can assume further that $F(i)=i$. Since the unit circle group of $\mathbf{C}$ acts transitively by inner automorphisms on the unit circle of the plane $j \mathbf{R}+k \mathbf{R}$ we can further assume that $F(j)=j$. Then we have either 
$F(k)=k$ or $F(k)=-k$. In the second case it is easy to see that $F$ is the map $\xi \mapsto k^{-1} \xi k$.

Instead of these arguments we could quote the paper [2] because it is obvious that these $F$ are orthogonal maps.

From now on we shall assume that $n \geq 2$. Let $a \in G, a \neq \pm e$. Then if we assume that $n \geq 3$ we deduce easily from Theorems $1-3$ that $\operatorname{dim}(a S \cap S)$ is maximal if and only if $a$ or $-a$ is a reflection, i.e., has the following eigenvalues

$$
\begin{aligned}
& +1 \text { with multiplicity } n-1, \\
& -1 \text { with multiplicity } 1 \text {. }
\end{aligned}
$$

This fact and (9) imply that if $a \in G$ is a reflection then either $F(a)$ or $-F(a)$ is again a reflection.

Now, let $e_{r}, 1 \leq r \leq n$ be the diagonal matrix whose $r$ th diagonal entry is -1 and all other diagonal entries are +1 . Then we have $e_{1}+\cdots+e_{n}=(n-2) e$, and

$$
F\left(e_{1}\right)+\cdots+F\left(e_{n}\right)=(n-2) e .
$$

Since (10) are the eigenvalues of $F\left(e_{r}\right)$ or of $-F\left(e_{r}\right)$ we have $\operatorname{Re} \operatorname{tr} F\left(e_{r}\right)$ $= \pm(n-2)$. From (11) we get $\sum_{r=1}^{n} \operatorname{Re} \operatorname{tr} F\left(e_{r}\right)=n(n-2)$. Hence, we must have $\operatorname{Re} \operatorname{tr} F\left(e_{r}\right)=n-2$ for every $r$. Thus $F\left(e_{r}\right)$ is a reflection for every $r$. By performing a similarity transformation by some matrix in $G$ we can assume that $F\left(e_{1}\right)=e_{1}$. The equation (11) now implies that $F\left(e_{2}\right), \ldots, F\left(e_{n}\right)$ each have 1 in the upper left corner. Since $F\left(e_{r}\right) \in G$ this must be the only nonzero entry of $F\left(e_{r}\right)$ lying in the first row or column. By continuing this argument we see that we can assume that $F\left(e_{r}\right)=e_{r}$ for $1 \leq r \leq n$.

Now, let $n=2$. If $D=\mathbf{R}$ then $S \cap G$ is invariant under $F$. Thus if $a=\left(\begin{array}{cc}0 & -1 \\ 1 & 0\end{array}\right)$ then either $F(a)=a$ or $F(a)=-a$. By composing $F$ with transposition we may assume that $F(a)=a$. Thus $F$ fixes every element of $\mathrm{SO}(2)$. Hence, $F\left(e_{1}\right)$ must be a reflection and we can assume as above that $F\left(e_{1}\right)=e_{1}$. Then, of course, $F\left(e_{2}\right)=e_{2}$ because $e_{2}=-e_{1}$.

If $D=\mathrm{C}, a \in G$ and $\operatorname{dim}(a \cdot S \cap S)=2$ then it follows from Theorem 2 that $a$ is either a reflection or else it is unitarily similar to a matrix $\left(\begin{array}{l}\lambda \\ 0\end{array}\right)$, where $\lambda \in \mathbf{C}$ is such that $|\lambda|=1$ and $\lambda \notin \mathbf{R}$. Thus (9) implies that $F\left(e_{1}\right)$ is either a reflection or else we can assume that $F\left(e_{1}\right)$ is the above matrix. But since $F\left(e_{1}\right) \in \operatorname{Re}+S, e_{1} \notin \operatorname{Re}+S$ and $\operatorname{Re}+S$ is $F$-invariant subspace it follows that the second case cannot occur. Hence, $F\left(e_{1}\right)$ is a reflection and we can assume that $F\left(e_{1}\right)=e_{1}$. Since $e_{2}=-e_{1}$ we will also have $F\left(e_{2}\right)=e_{2}$.

If $D=H, a \in G$ and $\operatorname{dim}(a \cdot S \cap S)=6$ then it follows from Theorem 3 that $a$ is either a reflection or else it is similar by some matrix in $G$ to a matrix $\lambda e$ where $\lambda \in \mathbf{H},|\lambda|=1$ but $\lambda \notin \mathbf{R}$. Again the latter case cannot occur because we can in that case assume that $F\left(e_{1}\right)=\lambda e$ but $\lambda e \in \mathbf{R} e+S, e_{1} \notin \mathbf{R} e+S$ and the subspace $\operatorname{Re}+S$ is $F$-invariant. It follows that $F\left(e_{1}\right)$ is a reflection and we can then assume that $F\left(e_{1}\right)=e_{1}, F\left(e_{2}\right)=e_{2}$. 


\section{Conjecture 6 (Orthogonal group).}

Theorem 4. Conjecture 6 is true when $G$ is the orthogonal group and $F$ is nonsingular.

Proof. We claim that if $u \in M_{n}(\mathbf{R})$ has rank 1 then also $F(u)$ has rank 1 .

Let $m_{p q}$ be the matrix whose $(p, q)$-entry is 1 and all other entries are zero. By performing a similarity transformation by an orthogonal matrix $a$ we can assume without loss of generality that $u=\alpha m_{11}+\beta m_{21}$. By performing another similarity transformation by $b \in G$ we can in addition assume that $F\left(e_{r}\right)=e_{n}$ $1 \leq r \leq n$.

Indeed, this reduction means that we are replacing $F$, which is supposed to satisfy $F(e)=e$, by the transformation $F_{1}$ defined as follows: $F_{1}(x)$ $=b F\left(a x a^{-1}\right) b^{-1}$.

It is easy to see that the subspace

$$
\left(\bigcap_{r=3}^{n} e_{r} \cdot S\right) \cap S
$$

has dimension 1 and the matrix $m_{12}-m_{21}$ lies in it. It follows from (8) that the subspace (12) is an invariant subspace of the linear transformation $F$. Thus $F\left(m_{12}-m_{21}\right)=\lambda\left(m_{12}-m_{21}\right)$. But $\left(m_{12}-m_{21}\right)+\frac{1}{2}\left(e_{1}+e_{2}\right) \in G$ and applying $F$ we get

$$
\lambda\left(m_{12}-m_{21}\right)+\frac{1}{2}\left(e_{1}+e_{2}\right) \in G .
$$

Therefore, $\lambda= \pm 1$.

Similarly, the subspace $\left(\cap_{r=3}^{n} e_{1} e_{r} S\right) \cap e_{1} S \cap e_{2} S$ is $F$-invariant, has dimension 1 and $m_{12}+m_{21}$ lies in it. Therefore,

$$
F\left(m_{12}+m_{21}\right)=\mu\left(m_{12}+m_{21}\right)
$$

and we get $\mu= \pm 1$ by a similar argument.

Since $u=\frac{1}{2} \alpha\left(e-e_{1}\right)+\frac{1}{2} \beta\left[\left(m_{21}-m_{12}\right)+\left(m_{21}+m_{12}\right)\right]$ we have

$$
F(u)=\frac{1}{2} \alpha\left(e-e_{1}\right)+\frac{1}{2} \beta\left[\lambda\left(m_{21}-m_{12}\right)+\mu\left(m_{21}+m_{12}\right)\right] .
$$

It follows that $F(u)$ has rank 1 because $\lambda= \pm 1$ and $\mu= \pm 1$.

Since $F$ preserves the matrices of rank 1 we must have (see [5]) either

$$
\begin{array}{ll}
F(x)=a x b & \text { for } x \in M_{n}(\mathbf{R}), \text { or } \\
F(x)=a^{t} x b & \text { for } x \in M_{n}(\mathbf{R}),
\end{array}
$$

where $a$ and $b$ are some nonsingular matrices. From $F(e)=e$ we deduce that $b=a^{-1}$. In both cases we must have $a y a^{-1} \in G$ for $y \in G$, i.e.,

$$
\left(a y a^{-1}\right) \cdot{ }^{t}\left(a y a^{-1}\right)=e, \quad \text { 'aay }=y^{t} a a .
$$


This implies that ' $a a=\rho e$ where $\rho>0$. Thus we have that $a \prime \sqrt{\rho} \in G$ which completes the proof.

Remark about the rotation group. It is obvious that the Conjecture 6 is also true for the case of the rotation group if $n$ is odd. Indeed, in that case $-e \notin \mathrm{SO}(n)$. If $F(\mathrm{SO}(n)) \subset \mathrm{SO}(n)$ and $x \in O(n)$ but $x \notin \mathrm{SO}(n)$ we have $-x=x \cdot(-e)$ $\in \mathrm{SO}(n)$ and $F(x)=-F(-x)=(-e) \cdot F(-x) \in O(n)$, i.e., $F(O(n)) \subset O(n)$. Thus, we can apply Theorem 4.

The Conjecture 6 is false for the rotation group if $n=2$. Indeed, in that case we can define $F$ as follows: $F$ fixes the matrices

$$
\left(\begin{array}{ll}
1 & 0 \\
0 & 1
\end{array}\right) \text { and }\left(\begin{array}{cc}
0 & -1 \\
1 & 0
\end{array}\right)
$$

and it maps

$$
\begin{aligned}
& \left(\begin{array}{cc}
1 & 0 \\
0 & -1
\end{array}\right) \mapsto\left(\begin{array}{ll}
1 & 0 \\
0 & 0
\end{array}\right) \\
& \left(\begin{array}{ll}
0 & 1 \\
1 & 0
\end{array}\right) \mapsto\left(\begin{array}{ll}
0 & 1 \\
0 & 0
\end{array}\right) .
\end{aligned}
$$

Then $F$ is nonsingular, it preserves $\mathrm{SO}(2)$ but not $O(2)$.

\section{Conjecture 6 (Unitary group).}

Theorem 5. Conjecture 6 is true when $G$ is the unitary group and $F$ is assumed to be nonsingular.

Proof. As shown in $\S 3$ we can assume that $F\left(e_{r}\right)=e_{r}$ for $1 \leq r \leq n$. Note that $F$ is assumed only to be R-linear map of $M_{n}(\mathrm{C})$ into itself. Our next objective is to reduce the problem to the case when $F$ is $\mathbf{C}$-linear.

We define the matrices $m_{p q}$ as in the previous section. The vector space $\left(\cap_{r=1}^{n} e_{r} S\right) \cap S$ is $F$-invariant and consists of diagonal matrices with purely imaginary diagonal entries. Thus, for instance,

$$
F\left(i m_{11}\right)=i \sum_{r=1}^{n} \alpha_{r} m_{r r}, \quad \alpha_{r} \in \mathbf{R} .
$$

But $e+(i-1) m_{11} \in G$ implies that

$$
e-m_{11}+i \sum_{r=1}^{n} \alpha_{r} m_{r r} \in G .
$$

Therefore, $\alpha_{1}= \pm 1$ and $\alpha_{r}=0$ for $2 \leq r \leq n$. Hence, in general,

$$
\begin{array}{ll}
F\left(\xi m_{r r}\right)=\xi m_{r r} & \text { for } \xi \in \mathbf{C}, \quad \text { or } \\
F\left(\xi m_{r r}\right)=\bar{\xi} m_{r r} & \text { for } \xi \in \mathbf{C},
\end{array}
$$

where, a priori, the alternative may depend on $r$. 
Assume that, say, we have $F\left(\xi m_{11}\right)=\xi m_{11}, F\left(\xi m_{22}\right)=\xi m_{22}$. Then by Theorem 2 we have

$$
\operatorname{dim}(a \cdot S \cap S)=2+(n-2)^{2} \text { and } \operatorname{dim}(F(a) \cdot S \cap S)=(n-2)^{2}
$$

where

$$
a=e+(i-1) m_{11}-(i+1) m_{22}, \quad F(a)=e+(i-1) m_{11}+(i-1) m_{22} .
$$

This contradicts Equation (9). Thus the alternative mentioned above is independent of $r$. By composing $F$ with conjugation we may assume that $F\left(\xi m_{r}\right)=\xi m_{r}$, $\xi \in \mathbf{C}, 1 \leq r \leq n$. Let, for instance, $\omega=\exp (i \pi / 4)$ and define $b=\omega e+$ $(i-\omega) m_{11}-(i+\omega) m_{22}$. Then $F(b)=b$ and the subspace $b \cdot S \cap S$ is $F$ invariant and consists of the matrices $\xi m_{12}-\bar{\xi} m_{21}, \xi \in \mathbf{C}$.

Since $e_{1} b \in G$ is diagonal we have $F\left(e_{1} b\right)=e_{1} b$. Thus the subspace $e_{1}(b \cdot S$ $\cap S)=\left(e_{1} b\right) \cdot S \cap e_{1} \cdot S$ is also $F$-invariant and consists of the matrices $\xi m_{12}+\bar{\xi} m_{21}, \xi \in \mathbf{C}$. Thus there exist real linear transformations $u_{1}, u_{2}: \mathbf{C} \rightarrow \mathbf{C}$ such that

$$
\begin{aligned}
& F\left(\xi m_{12}-\bar{\xi} m_{21}\right)=u_{1}(\xi) m_{12}-\overline{u_{1}(\xi)} m_{21}, \\
& F\left(\xi m_{12}+\bar{\xi} m_{21}\right)=u_{2}(\xi) m_{12}+\overline{u_{2}(\xi)} m_{21} .
\end{aligned}
$$

Hence,

$$
F\left(\xi m_{12}\right)=v_{1}(\xi) m_{12}+\overline{v_{2}(\xi)} m_{21}, \quad F\left(\bar{\xi} m_{21}\right)=v_{2}(\xi) m_{12}+\overline{v_{1}(\xi)} m_{21},
$$

where $2 v_{1}=u_{2}+u_{1}, 2 v_{2}=u_{2}-u_{1}$.

Since, for $|\lambda|=|\mu|=1, \lambda m_{12}+\bar{\mu} m_{21}+e-m_{11}-m_{22} \in G$, its image under $F$ is in $G$. This implies that $\left|v_{1}(\lambda)+v_{2}(\mu)\right|=1$ whenever $|\lambda|=|\mu|=1$. It follows that $v_{1}$ or $v_{2}$ is zero and the other one is an orthogonal transformation of $\mathbf{C}$.

Assume first that $v_{2}=0$. Then either $v_{1}(\xi)=\alpha \xi,|\alpha|=1$, or $v_{1}(\xi)=\alpha \bar{\xi}$, $|\alpha|=1$. In the first case we have $F\left(\xi m_{12}\right)=\xi F\left(m_{12}\right)$. Suppose that the second case is valid. Then we have $F\left(\xi m_{12}\right)=\alpha \bar{\xi} m_{12}, F\left(\xi m_{21}\right)=\bar{\alpha} \bar{\xi} m_{21}$. The quasidiagonal matrix having

$$
\left(\begin{array}{cc}
\cos \theta & i \sin \theta \\
\sin \theta & -i \cos \theta
\end{array}\right)
$$

in the upper left corner and ones on the remaining diagonal places is unitary. By applying $F$ we get a matrix which is not unitary if $\sin \theta \cos \theta \neq 0$. This is a contradiction.

Now, assume that $v_{1}=0$. Then either

$$
v_{2}(\xi)=\alpha \bar{\xi}, \quad|\alpha|=1, \quad \text { or } v_{2}(\xi)=\alpha \xi, \quad|\alpha|=1 .
$$

In the first case we have $F\left(\xi m_{12}\right)=\xi F\left(m_{12}\right)$. Suppose that the second case is valid. Then we have $F\left(\xi m_{12}\right)=\bar{\alpha} \bar{\xi} m_{21}, F\left(\xi m_{21}\right)=\alpha \bar{\xi} m_{12}$. By applying $F$ to the 
quasi-diagonal matrix mentioned above we get again a matrix which is not unitary if $\sin \theta \cos \theta \neq 0$. This is again a contradiction.

It follows that we must have $F\left(\xi m_{12}\right)=\xi F\left(m_{12}\right)$ for $\xi \in \mathbf{C}$.

Similarly $F\left(\xi m_{p q}\right)=\xi F\left(m_{p q}\right)$ for all $p, q$ and $\xi \in \mathbf{C}$, in other words $F$ must be C-linear.

Now, we can complete the proof as in the case of the orthogonal group, or else we can use the result of M. Marcus [3].

\section{Conjecture 6 (Symplectic Group).}

Theorem 6. Conjecture 6 is true when $G$ is the symplectic group and $F$ is assumed to be nonsingular.

Proof. From $\S 3$ we know that we can assume that $F\left(e_{r}\right)=e_{r}, 1 \leq r \leq n$. The subspace $\left(\cap_{r=1}^{n} e_{r} \cdot S\right) \cap S$ is $F$-invariant and consists of diagonal matrices with pure quaternionic entries. As in the case of the unitary group we obtain that $F\left(\xi e_{r}\right)=\sigma_{r}(\xi) e_{r} 1 \leq r \leq n, \xi \in \mathbf{H}$, where $\sigma_{r} \in A$. For every $r$ there exists a quaternion $\alpha_{r}$ of unit norm such that

$$
\sigma_{r}(\xi)=\alpha_{r} \xi \alpha_{r}^{-1}=\alpha_{r} \xi \bar{\alpha}_{r}, \quad \xi \in \mathbf{H} .
$$

Let $a$ be the symplectic diagonal matrix with diagonal entries $\alpha_{1}, \ldots, \alpha_{n}$. Then replacing $F$ by $F_{1}$ which is defined as follows $F_{1}(x)=F\left(a x a^{*}\right)$ we can assume that $F$, in addition, satisfies $F\left(\xi e_{r}\right)=\xi e_{n} 1 \leq r \leq n$, for all $\xi \in \mathbf{H}$.

Let $S^{\prime}=S \cap M_{n}(\mathbf{R})$ be the space of real skew-symmetric matrices and $S^{\prime \prime}$ the space of real symmetric matrices. Then $S$ is a direct sum

$$
S=S^{\prime}+i S^{\prime \prime}+j S^{\prime \prime}+k S^{\prime \prime}
$$

where $1, i, j, k$ are the standard units of $\mathbf{H}$. It is obvious that

$$
\begin{aligned}
S^{\prime \prime} & =i S \cap j S \cap k S, & i S^{\prime \prime} & =S \cap j S \cap k S, \\
j S^{\prime \prime} & =S \cap i S \cap k S, & k S^{\prime \prime} & =S \cap i S \cap j S .
\end{aligned}
$$

Since, say, $i \cdot S=(i e) \cdot S$ and $i e \in G, F(i e)=i e$ then we can use formula (8) to get

$$
F\left(S^{\prime \prime}\right)=S^{\prime \prime}, \quad F\left(i S^{\prime \prime}\right)=i S^{\prime \prime}, \quad F\left(j S^{\prime \prime}\right)=j S^{\prime \prime}, \quad F\left(k S^{\prime \prime}\right)=k S^{\prime \prime} .
$$

The space

$$
\left(\bigcap_{r=3}^{n} e_{r} \cdot S\right) \cap S
$$

consists of skew-hermitian matrices whose nonzero entries occur only on the diagonal or at places $(1,2),(2,1)$. We shall use the fact that this space is $F$ invariant.

Let $|\xi|=1$ and $\left|\xi_{r}\right|=1,3 \leq r \leq n$ where $\xi, \xi_{r} \in \mathbf{H}$. Then 


$$
\xi m_{12}-\bar{\xi} m_{21}+\xi_{3} m_{33}+\cdots+\xi_{n} m_{n n} \in G
$$

and by applying $F$ we get

$$
F\left(\xi m_{12}-\bar{\xi} m_{21}\right)+\xi_{3} m_{33}+\cdots+\xi_{n} m_{n n} \in G .
$$

Now, the fact that the above space is $F$-invariant implies that the nonzero entries of $F\left(\xi m_{12}-\bar{\xi} m_{21}\right)$ are concentrated in the $2 \times 2$ block in the upper left corner of that matrix and that this block is a symplectic matrix.

Let

$$
\begin{aligned}
& b=(i-1) m_{11}-(i+1) m_{22}+e, \\
& c=(j-1) m_{11}-(j+1) m_{22}+e, \\
& d=(k-1) m_{11}-(k+1) m_{22}+e .
\end{aligned}
$$

Then $b, c, d \in G$ are diagonal, $F(b)=b, F(c)=c, F(d)=d$ and the space

$$
b \cdot S \cap c \cdot S \cap d \cdot S
$$

is $F$-invariant. The intersection of the spaces (13) and (14) consists of the matrices of the form

$$
\left(\begin{array}{ccccc}
0 & \alpha & & & \\
-\alpha & 0 & & & \\
& & \xi_{3} & & \\
& & & \ddots & \\
& & & & \xi_{n}
\end{array}\right), \quad \bar{\xi}_{r}=-\xi_{r},
$$

where $\alpha$ is real. Therefore, $F\left(m_{12}-m_{21}\right)= \pm\left(m_{12}-m_{21}\right)$.

In general, $F\left(m_{p q}-m_{q p}\right)= \pm\left(m_{p q}-m_{q p}\right)$ which means that $F\left(S^{\prime}\right)=S^{\prime}$. Since also $F\left(S^{\prime \prime}\right)=S^{\prime \prime}$ we conclude that $M_{n}(R)$ is $F$-invariant. Thus $F$ preserves $O(n)$ and by Theorem 4 we may assume, in addition to other properties of $F$, that $F$ is identity on $M_{n}(\mathbf{R})$.

Let us multiply on the left by $i$ each of the subspaces $e_{r} \cdot S, S, b \cdot S, c \cdot S, d \cdot S$ which occur in (13) and (14). Then by taking their intersection we get an $F$ invariant subspace which consists of the matrices $i x$ where $x$ has form (15). Hence we have

$$
F\left(i m_{12}-i m_{21}\right)=\lambda i\left(m_{12}-m_{21}\right)+i\left(\eta_{3} m_{33}+\cdots+\eta_{n} m_{n n}\right)
$$

where $\lambda \in \mathbf{R}$ and $\bar{\eta}_{r}=-\eta_{r}$.

If $\left|\xi_{r}\right|=1$ for $3 \leq r \leq n$ then

$$
i\left(m_{12}-m_{21}\right)+i\left(\xi_{3} m_{33}+\cdots+\xi_{n} m_{n n}\right) \in G
$$

and also its image by $F$ is in $G$. This implies that $\lambda= \pm 1$ and $\eta_{r}=0$ for $3 \leq r \leq n$. Hence, 


$$
F\left(i\left(m_{12}-m_{21}\right)\right)= \pm i\left(m_{12}-m_{21}\right)
$$

and similarly,

$$
F\left(i\left(m_{p q}-m_{q p}\right)\right)= \pm i\left(m_{p q}-m_{q p}\right) .
$$

Thus $F\left(i S^{\prime}\right)=i S^{\prime}$ and by similar arguments $F\left(j S^{\prime}\right)=j S^{\prime}, F\left(k S^{\prime}\right)=k S^{\prime}$. It follows that the subspaces $i M_{n}(\mathbf{R}), j M_{n}(\mathbf{R}), k M_{n}(\mathbf{R})$ are also $F$-invariant. Thus $F$ maps $U(n)$ onto itself and now by Theorem 5 we can conclude that the restriction of $F$ to the subspace $M_{n}(\mathbf{R})+i M_{n}(\mathrm{R})$ is orthogonal with respect to the scalar product (3). Using two other embeddings of $\mathbf{C}$ into $\mathbf{H}$ we see that also the restrictions of $F$ to the subspaces $M_{n}(\mathbf{R})+j M_{n}(\mathbf{R})$ and $M_{n}(\mathbf{R})+k M_{n}(\mathbf{R})$ are orthogonal. This implies that the linear transformation $F$ is orthogonal for the same scalar product. Hence we can now use the result [2] to complete the proof.

\section{Conjecture 7.}

Theorem 7. Conjecture 7 is true.

Proof. Let $x, y, z, t \in V$. Then we have

$$
\langle u((x+y) \otimes z), u((x+y) \otimes z)\rangle=\langle(x+y) \otimes z,(x+y) \otimes z\rangle,
$$

$\langle u(x \otimes z)+u(y \otimes z), u(x \otimes z)+u(y \otimes z)\rangle=\langle x \otimes z+y \otimes z, x \otimes z+y \otimes z\rangle$, $\langle u(x \otimes z), u(y \otimes z)\rangle+\langle u(y \otimes z), u(x \otimes z)\rangle=\langle x \otimes z, y \otimes z\rangle+\langle y \otimes z, x \otimes z\rangle$,

$$
\operatorname{Re}\langle u(x \otimes z), u(y \otimes z)\rangle=\operatorname{Re}\langle x \otimes z, y \otimes z\rangle .
$$

By replacing $x$ with $i x$ we get also

$$
\operatorname{Im}\langle u(x \otimes z), u(y \otimes z)\rangle=\operatorname{Im}\langle x \otimes z, y \otimes z\rangle .
$$

Thus $\langle u(x \otimes z), u(y \otimes z)\rangle=\langle x \otimes z, y \otimes z\rangle$. Now, replace $z$ by $z+t$. Then we get

$$
\begin{aligned}
& \langle u(x \otimes z)+u(x \otimes t), u(y \otimes z)+u(y \otimes t)\rangle=\langle x \otimes z+x \otimes t, y \otimes z+y \otimes t\rangle, \\
& \langle u(x \otimes z), u(y \otimes t)\rangle+\langle u(x \otimes t), u(y \otimes z)\rangle=\langle x \otimes z, y \otimes t\rangle+\langle x \otimes t, y \otimes z\rangle .
\end{aligned}
$$

Replacing $z$ by $i z$ we get

$$
\langle u(x \otimes z), u(y \otimes t)\rangle-\langle u(x \otimes t), u(y \otimes z)\rangle=\langle x \otimes z, y \otimes t\rangle-\langle x \otimes t, y \otimes z\rangle .
$$

Therefore, $\langle u(x \otimes z), u(y \otimes t)\rangle=\langle x \otimes z, y \otimes t\rangle$ which means that $u$ is unitary.

Acknowledgment. I would like to thank Dr. Y. H. Au-Yeung for his careful reading of the manuscript and several corrections.

\section{REFERENCES}

1. N. Bourbaki, Eléments de mathématique. XXIII. Part. 1. Les structures fondamentales de l'analyse. Livre II: Algèbre. Chap. 8: Modules et anneaux semisimples, Actualités Sci. Indust., no. 1261, Hermann, Paris, 1958. MR 20 \#4576. 
2. D. Ż. Djoković, A characterization of minimal left or right ideals of matrix algebras, Linear and Multilinear Algebra 1 (1973), 139-147.

3. M. Marcus, All linear operators leaving the unitary group invariant, Duke Math. J. 26 (1959), 155-163. MR 21\#54.

4. - Linear transformations on matrices, J. Res. Nat. Bur. Standards 75 B (1971), 107-113.

5. R. Westwick, Transformations on tensor spaces, Pacific J. Math. 23 (1967), 613-620. MR 37 \#1397.

DEPARTMENT OF PURE MATHEMATICS, UNIVERSITY OF WATERLOO, WATERLOO, ONTARIO, CANADA 